\title{
Cross-sectional study on sensitization to mite and cockroach allergen components in allergy patients in the Central European region
}

\author{
Petr Panzner ${ }^{1 *} \mathbb{C}$, Martina Vachová ${ }^{1}$, Tomáš Vlas ${ }^{2}$, Petra Vítovcová ${ }^{2}$, Petra Brodská ${ }^{3}$ and Marek Malý ${ }^{4}$
}

\begin{abstract}
Background: The major sources of allergens in the indoor air include house dust mites, dander derived from domestic animals and rodents, cockroach, and several fungi. Mites are the main cause of allergies in some countries with a warmer climate, but the epidemiological significance of mite and cockroach allergens in Central Europe has not been established yet.
\end{abstract}

Methods: We assessed sensitization profiles of allergy patients in a Central European region in regard to sensitization to mites and cockroach. We used molecular diagnosis by means of the microarray ISAC, and we investigated 1766 patients with clinical suspicion to an allergic disorder. 1255 of them were positive to at least one allergen component, and this group was subjected to statistical analysis.

Results: The sensitization to at least one mite-specific molecule (Der $p 1$, 2, Der f 1,2) was observed relatively frequently in $32.7 \%$ of patients. Specific IgE to mite group 2 molecules is almost fully cross-reactive. Group 1 allergens are also cross-reactive, but in some patients, a species-specific response was observed. Relatively high rate of sensitization both to group 1 and 2 allergens in our patients indicates the greater role of co-sensitizations. Isolated sensitizations to molecules derived from glyciphagid mites Lep d 2 and/or Blo t 5 without sensitization to other mite-derived molecules were observed only exceptionally (in $0.6 \%$ of cases). True sensitization to at least one cockroach-specific molecule (Bla g 1,2,5) was very rare (in $0.6 \%$ of cases), and nearly all of them were co-sensitizations with other noncockroach-derived molecules. Sensitization to an inhaled tropomyosin was observed rarely in $2.2 \%$ of patients (Der p 10 in 1.9\% and Bla g 7 in 1.5\%). Co-sensitization of inhaled tropomyosins with the respective mite- or cockroach-specific molecules was observed only in the minority of patients suggesting the different route of sensitization being more frequent.

Conclusions: The majority of patients are co-sensitized to several molecules of the respective allergen source. The knowledge of this molecular spectrum of sensitization is important for optimal diagnosis and treatment in respect to allergen content in mite extracts used for diagnostic and therapeutic purposes. In regard to the sensitization patterns of Central European patients, it is necessary to point out the importance of quantifying at least three major mite components Derf 1, Der $p 1$ and Derf 2 (or Der p 2).

\footnotetext{
*Correspondence: panzner@fnplzen.cz

${ }^{1}$ Department of Immunology and Allergology, Faculty of Medicine

in Pilsen, Charles University, Pilsen, Czech Republic

Full list of author information is available at the end of the article
} 


\section{Background}

Immediate hypersensitivity to indoor allergens is a risk factor for asthma, and allergic rhinitis and sensitization to these allergens may play a role in atopic dermatitis as well. The major sources of allergens in the indoor air include house dust mite (HDM), dander derived from domestic animals and rodents, cockroach, and several fungi. Given that most persons in Western societies spend more than $90 \%$ of their lives in indoor environments, it is not surprising that indoor allergens play an important role in allergic sensitization and symptoms. Sensitization to distinct molecules may represent higher risk for asthma, or atopic dermatitis $[1,2]$ and several studies suggest that sensitization to multiple molecules ("molecular spreading") is associated with a higher probability of more severe symptoms of allergy [2-4].

HDM is the main cause of allergies in some countries with a warmer climate [5]; in Central Europe, the sensitization rates to mites, some animals (especially cats and dogs) and molds (especially Alternaria) immediately follow the sensitization rates to pollens $[6,7]$. Cockroach allergy is an important cause of asthma in several regions of America and Asia [8], its significance in Central Europe has not been established yet.

While the diagnosis of immunoglobulin $\mathrm{E}$ (IgE)-mediated inhalant allergy is primarily based on clinical history and sensitization, that is demonstrated via skin prick testing and measurement of serum allergen-specific IgE; this methodology has its limitations. In vitro and in vivo allergy testing are often based on, the insufficiency of standardized allergen extracts owing to the natural variability of the allergen source, or manufacturing procedure, can differ regarding their allergenic content. This issue was already confirmed also for HDM allergens [9-11]. An even more important disadvantage of allergenic extracts is that they are incapable of differentiating between primary sensitization and immunological cross-reactivity in multiple sensitizations which are observed in many patients. Nonetheless, natural allergenic extracts were the cornerstone of inhalant allergy diagnosis until several years ago, when the molecular diagnosis was made possible by advances in molecular biology which lead to the development of a large spectrum of purified natural and recombinant allergenic molecules. Such presently routinely available reagents enable the use of the diagnostic approach commonly known as a component-resolved diagnosis of allergy, and now allow the systematic study of the principal allergens and cross-reactivity processes involved in allergic sensitization.

The introduction of microarrays with a much larger number of purified or recombinant molecules constituted a further development in the diagnosis of allergic diseases. Such microarrays now represent powerful tools in the screening of serum IgE-reactivity, and they allow the definition of sensitization profiles. Identification of sensitizations and co-sensitizations to species-specific and cross-reacting allergen components may be especially important in decisions concerning allergen-specific immunotherapy.

This study aimed to assess the usefulness of molecular diagnosis using a microarray in the description of sensitization profiles in subjects showing a sensitization to HDM and cockroach living in the Central European region, with a special focus on discriminating between cross-reactivities and multiple sensitizations to different allergens. Although not much data on the diagnostic accuracy of the microarray ImmunoCAP ISAC in HDM and cockroach allergy is available, we decided to use this approach because of the possibility it provides to analyze a wide spectrum of component sensitizations. Furthermore, some studies have shown similar performances for component-based microarray ISAC and whole-allergen CAP system detection $[12,13]$. Nonetheless, it is necessary to bear in mind the possible different sensitivities to individual molecules in the used assay.

\section{Methods}

This cross-sectional observational study was conducted according to the STROBE recommendations [14] to the extent which may apply to this study design. We retrospectively analyzed data from 1766 patients who had been examined in the years 2011-2014 based on suspicion of allergy at the outpatient service of the Department of Immunology and Allergology of the University Hospital in Pilsen; the patients came from the western part of the Czech Republic. One thousand two hundred fifty-five patients positive to at least one allergen component were subjected to further detailed analysis. This test group of 1255 sensitized patients had at least one of the following diagnoses: chronic rhinitis (73\%), bronchial asthma (41\%), atopic dermatitis (34\%), urticaria or edema (19\%), and/or anaphylaxis (11\%). Patient ages ranged from 1 to 68 years, with a mean age of 29 years. The sex ratio was $45.3 \%$ men to $54.7 \%$ women.

The detection of specific IgE to multiple allergen components was performed using the 112 component ImmunoCAP ISAC allergen microarray immunoassay (Thermo Fisher Scientific, Uppsala, Sweden). Briefly, microarray reaction sites were incubated with $20 \mu \mathrm{l}$ undiluted patient serum for $2 \mathrm{~h}$ to capture allergen-specific IgE antibodies by their corresponding allergen. Subsequently, the microarray slides were rinsed and washed to remove unbound sIgE. After drying, complexes of allergen-bound sIgE were stained with a secondary, fluorescence-labeled antihuman IgE for $1 \mathrm{~h}$ at room temperature while protected from light. After a second rinsing and washing procedure, 
the obtained fluorescence signals were scanned using a laser scanner (LuxScan 10K; CapitalBio, Beijing, China). Analysis of the corresponding digitized microarray images was performed using ImmunoCAP ISAC software, and image information was transformed into numerical data according to a reference serum of known IgE content. Results were expressed as ISAC standardized units (ISU), and values greater than or equal to 0,3 ISU/l were taken as positive.

The analysis was focused on inhalant mite- and cockroach-derived specific allergen components and potentially cross-reactive components which are included in the ISAC system. Specific allergy markers are represented by the group 1 (nDer p 1 , nDer $\mathrm{f} 1$ ), group 2 (rDer $\mathrm{p} 2$, rDer f 2, rLep d 2) and group 5/21 (rBlo t 5 ) allergens for mites, and molecule rBla g 1 , aspartic protease rBla g 2 and glutathione-S-transferase rBla g 5 for cockroach. Finally, panallergens like mite- and cockroach-derived tropomyosins (rDer p 10 and nBla g 7) were also included in the analysis and related to sensitizations to other tropomyosins (nPen m 1 and rAni s 3 ).

\section{Results}

The results of the analysis describing the HDM and cockroach sensitization patterns in the group of 1255 patients sensitized to at least one allergen component in our region are listed below. All percentages were calculated using the whole group of 1255 patients.

\section{Mites}

The sensitization to at least one mite-specific molecule (Der p 1, 2, Der f 1, 2) was observed in $32.7 \%$ of patients. Isolated sensitizations to molecules derived from glyciphagid mites Lep d 2 and/or Blo t 5 without sensitization to other mite-derived molecules were observed only in $0.6 \%$ of cases.

\section{Cockroach}

True sensitization to at least one cockroach-specific molecule (Bla g $1,2,5$ ) was very rare (in $0.6 \%$ of cases), and nearly all of them were co-sensitizations with other noncockroach-derived molecules, including mite-derived molecules in half of the patients (4 cases).

\section{Tropomyosins}

Sensitization to an inhaled mite- and cockroach-derived tropomyosin was observed in $2.2 \%$ of patients (Der p 10 in $1.9 \%$ and Bla $g$ in $1.5 \%$ ).

Co-sensitization of Der p 10 with other mite-derived molecules was observed in $0.7 \%$ of patients. Co-sensitization of Bla $\mathrm{g} 7$ with other cockroach-specific molecules was exceptional (in 0.1\%), without co-sensitization to Der p 10 in all cases.
In patients sensitized to Der p 10 and not to Bla g 7 $(0.6 \%)$ no sensitization to other tropomyosins (Pen $\mathrm{m} 1$, Ani s 3) was observed in nearly all of the cases ( $0.5 \%)$, and sensitization to other mite-derived molecules was observed only in $0.2 \%$ of cases. In patients sensitized both to Der p 10 and Bla g 7 (1.3\%), sensitization to other tropomyosins was observed in all cases, and sensitization to other mite-derived molecules was observed in $0.5 \%$ of cases. Monosensitization (in the frame of tropomyosins) to Bla g 7 was only exceptional.

The frequency of sensitization to individual mitederived molecules, and their co-sensitizations is shown in Figs. 1 and 2. Sensitizations and co-sensitizations in the frame of tropomyosins and mites are shown in Fig. 3. The sensitization to mite-specific molecules in the context of sensitizations to molecules specific for other inhalant allergens is shown in Fig. 4.

\section{Discussion}

The observed sensitization rate to perennial inhalantderived molecules in our group of patients is lower than the sensitization rate to pollen-derived components [6]. However, the relatively high sensitization rate to mites underlines their clinical importance in the Central European region. It needs to be emphasized that in this paper we focus only on sensitization rates and not their clinical relevance; carrying out the latter analysis without using specific provocation tests might become rather complicated and is not realistic in such large cohorts.

\section{Cockroach}

It has been suggested that a cocktail of five allergens Bla g 1 and/or Per a 1, Bla g 2, Bla g 4, Bla g 5, and Bla g 7 and/or Per a 7 would be expected to diagnose $50-64 \%$ of cockroach-allergic patients $[15,16]$. The degree of homology between the lipocalin Bla g 4 and the mammalian lipocalins is low, and only small cross-reactivity with these mammalian allergens would be expected [8]. We did not analyze this cross-reactivity because of the very low rate of sensitization to Bla g 4 in our patients. The low rate of sensitization to cockroach-derived molecules was caused probably rather by the low presence of cockroaches in our climate than by missing molecules in the used assay.

\section{Mites}

According to several studies, HDM sensitization and allergy are considered to be the most frequent among inhalant allergies $[5,17,18]$. In our conditions, it holds the fourth position behind grass pollen (sensitization frequency for Phl p 1 is $60.8 \%$ ), birch pollen (sensitization frequency for Bet $v 1$ is $47.3 \%$ ), and cat allergens (sensitization frequency for Fel d 1 is 31.5\%) [6], what differs 


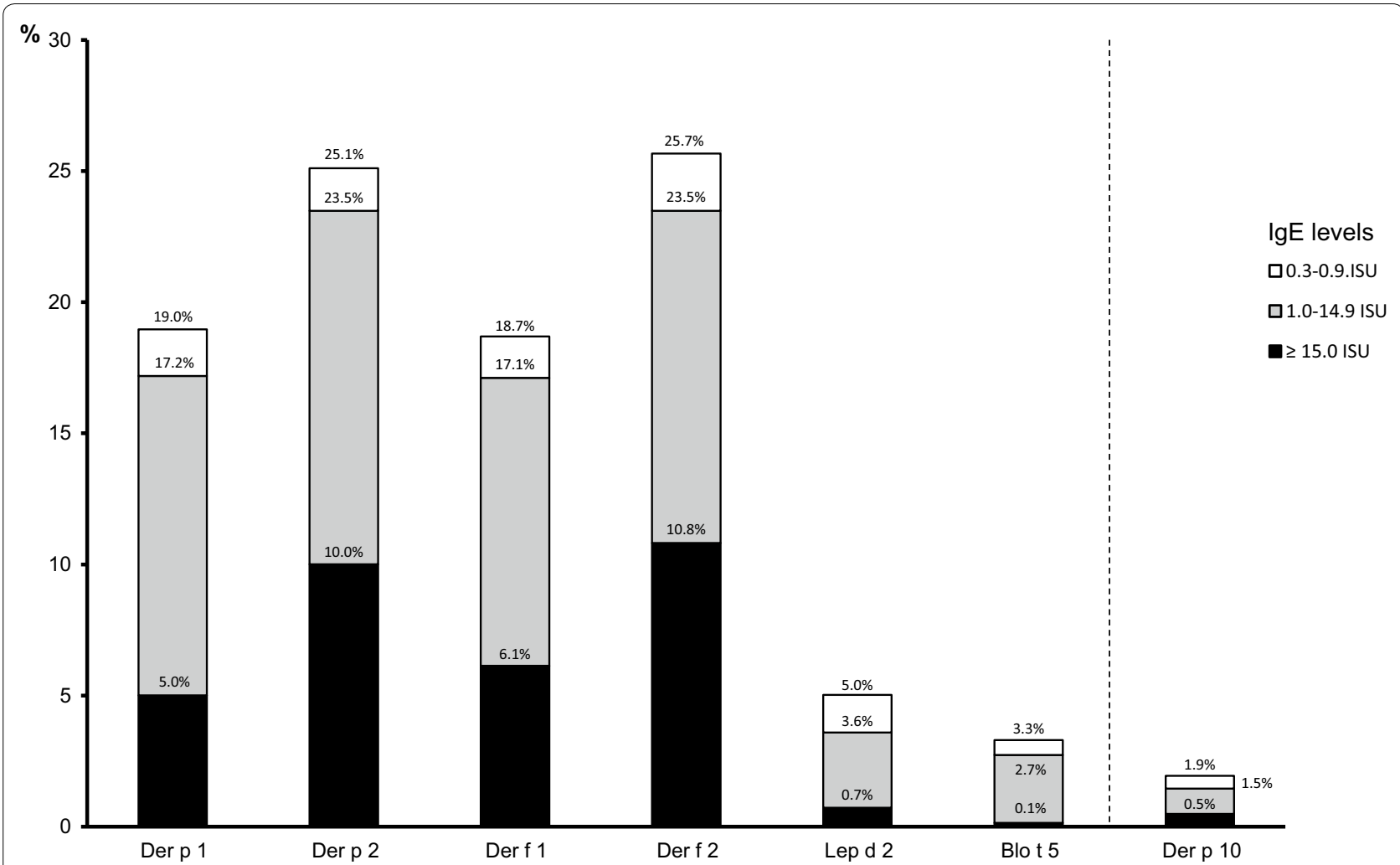

Fig. 1 Sensitization rates to mite-derived molecules

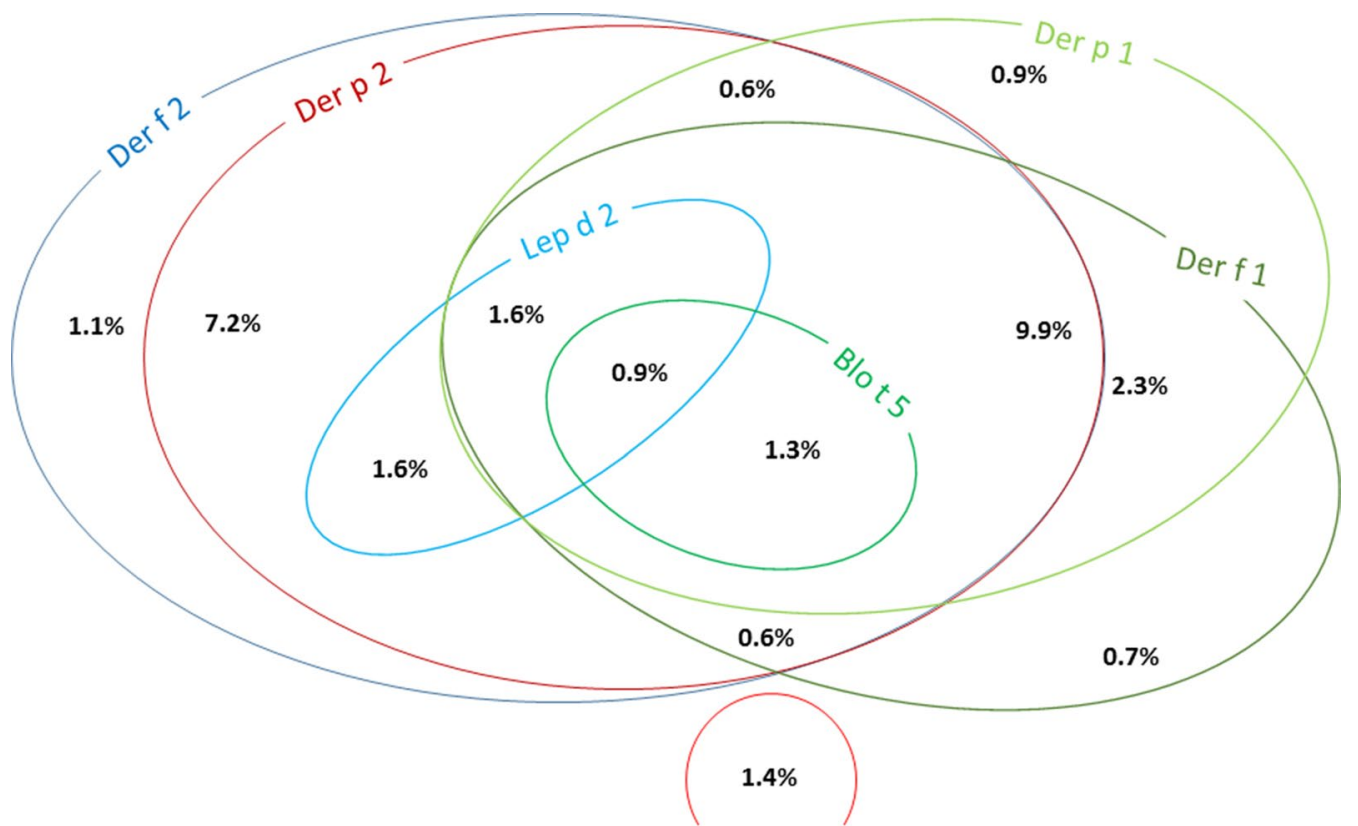

Der $\mathrm{p} 10$

Fig. 2 Venn diagram depicting mono- and co-sensitizations to mite-derived molecules. Mono- and co-sensitizations with a frequency of less than $0.6 \%$ are not shown 


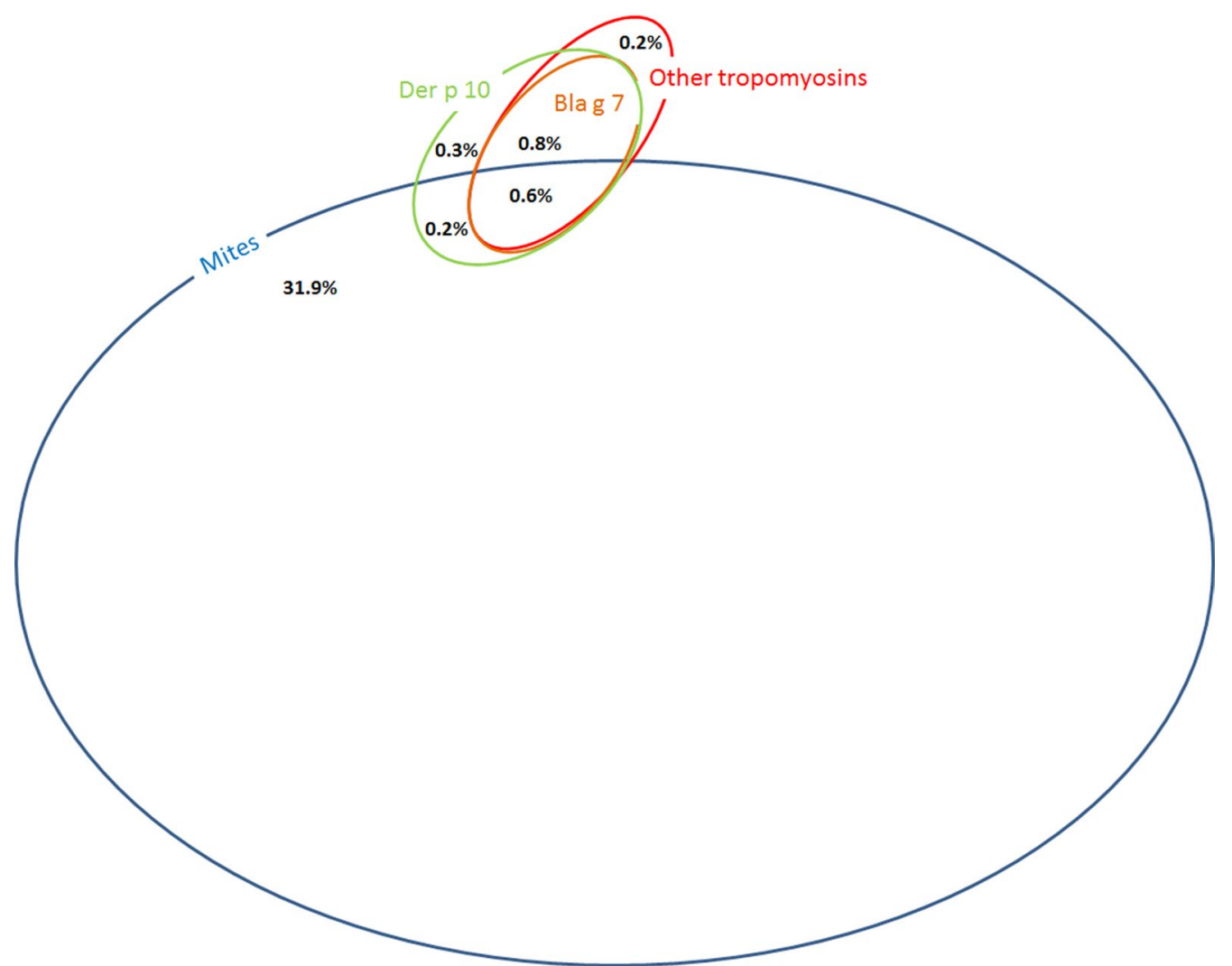

Fig. 3 Venn diagram depicting mono- and co-sensitizations to tropomyosins in relation to sensitization to mites ( $=$ sensitization to at least one mite-specific molecule). Mono- and co-sensitizations with a frequency of less than $0.2 \%$ are not shown

from data coming from other regions $[17,19,20]$. This discrepancy may be caused by geographical differences or may be due to the selected population, mainly consisting of adults with predominant respiratory allergy. Higher sensitization rate to grass pollen in adolescents was also shown in a study from northern Italy while the leading position of mites was pronounced clearly in smaller children [19].

It is known that patients sensitized to mites are not always sensitized to the molecules used in our study, other molecules may also play a role, but the number of patients sensitized only to these other molecules is generally very low [21-25]. Moreover, cysteine protease (Der p 1, Der f 1) signaling has been described to have a strong TH2 up-regulation effect, and group 2 allergens have been shown to bind TLR4 via binding LPS, thus having a stronger immunogenic potential further enhancing the complexity of mite allergy. Hence, these molecules may play a leading role in the atopic march, and a differential sensitization rate in children compared to adults may be present [26]. Sensitization to group 1 allergens was shown to be more frequent in children [18], thereby suggesting a possible role of group 1 allergens in the onset of sensitization, perhaps mediated by their proteolytic activity and direct epithelial damage [27]. The more frequent sensitization to group 2 allergens in the adult population suggested a later sensitization to group 1 allergens what was documented by observation of a birth cohort [26]. The ability of group 2 allergens to bind directly to TLR4 might be an explanation for this observation. These facts may also explain that in the case of mites the higher the exposure, the more severe the clinical allergic condition, in contrast to cat allergens where overexposure seems to lead to tolerance [28].

Dermatophagoides pteronyssinus is the most widespread mite all over the world; it predominates especially in humid regions where the climate is more influenced by the ocean. Dermatophagoides farinae is supposed to occur more in the continental regions of Europe and the Mediterranean area, but most countries have mixed populations $[22,29]$. In our patients, sensitization to $D$. pteronyssinus and $D$. farinae was almost equal in contrast to Spain where the IgE-prevalence to $D$. pteronyssinus allergens was found to be slightly higher than to $D$. farinae allergens [18]. 


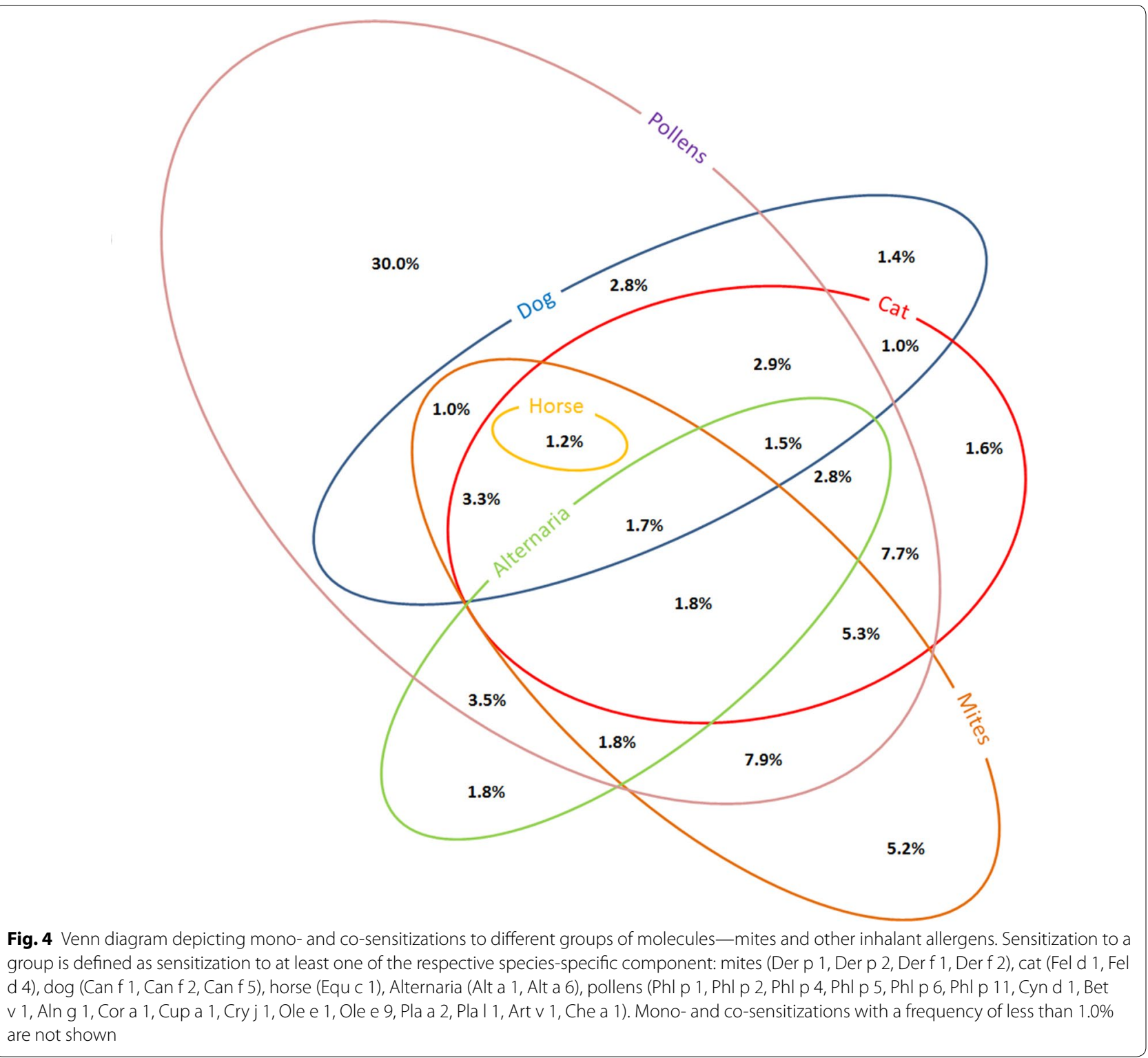

Although the pyroglyphid HDM D. pteronyssinus and $D$. farinae seem to predominate, glyciphagid mites may also be important in some regions [18, 21,29].

Blomia tropicalis is the most important house dust mite from the family glycyphagidae. It is most abundant in tropical regions $[4,29]$. The major allergen Blo t 5 shows $40 \%$ sequence homology with Der p 5, but it was reported not to cross-react with each other [29]. As B. tropicalis does not form part of the acarofauna in Central Europe, the not negligible sensitization rate in our patients seems to be attributable rather to potential cross-reactivity among allergens produced by different mite species (mite group 5/21 molecules) than to true sensitization. This assumption is also supported by low levels of sensitization to Blo t 5 in our group. The results of another study suggested that allergens different from those belonging to group 5 may also be responsible for the partial cross-reactivity among different mite species [30].

Lepidoglyphus destructor, a glycyphagid storage mite, may also become a HDM [29]. Sensitization from domestic exposure was reported from Sweden and France. It is not possible to decide whether positivity to Lep $\mathrm{d} 2$ in our patients was due to true sensitization or cross-reactivity within mite group 2 molecules. The absence of monosensitizations to Lep d 2 (in the frame of mite-derived molecules) in our patients testifies rather for Lep $d 2$ sensitization due to cross-reactivity with other group 2 
allergens, what is in contradiction to the data suggesting that no such cross-reactivity exists [18].

Mite sensitized patients in our group were usually cosensitized to several mite-specific components; monosensitization was markedly less frequent (Fig. 2). Der $f$ 1 and Der $\mathrm{p} 1$ (cysteine proteases) and Der $\mathrm{f} 2$ and Der p 2 (lipid binding proteins) are assumed to be the specific components most commonly involved in mite allergy. The predominant sensitization, both regarding prevalence and intensity, to mite group 2 molecules was already described $[17,18,25]$ and confirmed by our results. In a French study, predominant sensitization to Der p 1 (93\%) was detected [31], and we may speculate that this difference might be due to the different population studied. The same French study also detected a higher frequency of sensitization to Der p 10 (28\%) signaling the primary sensitizing agent possibly being shrimp which is much more consumed in the Mediterranean area than in Central Europe.

Tropomyosins are molecules responsible for crossreactivity among mites, shrimp, and cockroach [32]. IgE binding to the mite group 10 allergens is rare in Europe [21] and Australia [33] and, from one study, in US subjects allergic to both HDM and cockroach [34]. Similarly, in this study's population group, sensitization to individual tropomyosins was considerably less frequent than sensitization to more tropomyosin molecules together suggesting present cross-reactivity in the frame of this group of molecules (Fig. 3).

Sensitization to the tropomyosin Der p 10 was not observed frequently (in $1.9 \%$ ) in our patients, and cosensitizations with other mite-specific molecules were not regular (in $0.8 \%$ ) suggesting the possible different route of sensitization in a considerable proportion of the patients. Tropomyosins represent clinically relevant seafood allergens, but the role of mite tropomyosin, Der p 10, in house dust mite allergy has not been studied in detail. A hypothesis that tropomyosin sensitization may indicate a true food allergy independent of mite respiratory disease has been proposed [18]. Another hypothesis pretends that Der p 10 may be a diagnostic marker for mite-allergic patients with additional sensitization to allergens other than Der p 1 and Der p 2. Such patients may require attention when allergen-specific immunotherapy is considered [35].

The high rate of simultaneous sensitizations to different mite components (Fig. 2) may be explained either by co-sensitizations or by cross-reactivities. Specific IgE to Der $\mathrm{p} 2 /$ Der $\mathrm{f} 2$ is almost fully cross-reactive, but no cross-reactivity was described with Lep d 2 [18]. Group 1 allergens are also cross-reactive, but in some patients, a species-specific response was observed [18]. Relatively high rate of sensitization both to group 1 and 2 allergens in our patients indicates the greater role of co-sensitizations.

\section{Conclusions}

The vast majority of mite-sensitized patients in our group showed polysensitization to two or more components derived from both major HDM, i.e., D. pteronyssinus and $D$. farinae. On the contrary, only a minority was sensitized to mite-derived tropomyosin, and these sensitizations were not frequently connected to sensitization to other mite-derived molecules, suggesting dominating different route of sensitization via food-derived tropomyosins.

It is necessary to stress the importance of the knowledge of allergen content in mite extracts used for diagnostic and therapeutic purposes as a practical implication from this study. Several studies have focused on this issue and analyzed the composition of several commercially available preparations concerning their qualitative and quantitative allergen composition. There are evident considerable differences in the allergen content among the commercially available extracts [9-11]. Although some authors question the necessity of tailoring of allergen immunotherapy to the sensitizing species [22], we consider this to be important for the optimal efficacy of this treatment. Group 2 allergens are highly cross-reactive, but as group 1 sensitization could be species specific in some patients and its prevalence is higher in children, an adequate balance of major mite species and major allergens must be considered in the design of mite allergy vaccines. Regarding the sensitization patterns of patients within the Central European region, it is necessary to point out the importance of quantifying at least three major mite components Der $\mathrm{f} 1$, Der $\mathrm{p}$ 1 and Der $\mathrm{f} 2$ (or Der p 2). Such information is crucial for effective diagnosis and treatment. Besides these molecules, Der p 23, a new major house dust mite allergen, should be considered to be an important component for allergen-specific immunotherapy as well [26, 36, 37]. The importance of eventual further allergens (so-called "midtier" allergens-e.g., Der p 5) in this context has yet to be elucidated. There is an urgent need for rigorous, longterm clinical trials with an efficacy criterion to find the consensus on the dose of individual molecules in HDMallergen-specific immunotherapy [37, 38].

\section{Abbreviations}

HDM: house dust mite; IgE: immunoglobulin E; ISAC: immuno solid-phase allergen chip; STROBE: strengthening the reporting of observational studies in epidemiology; LPS: lipopolysaccharide.

\section{Authors' contributions}

PP participated in study design, data analysis, and manuscript writing. MV participated in study design, data analysis, and manuscript writing. TV 
participated in laboratory analyses, anonymization of the data, and critical revision of the manuscript. PV participated in data analysis and critical revision of the manuscript. PB participated in data analysis and critical revision of the manuscript. MM participated in data analysis, statistical calculations, and critical revision of the manuscript. All authors read and approved the final manuscript.

\section{Author details}

1 Department of Immunology and Allergology, Faculty of Medicine in Pilsen, Charles University, Pilsen, Czech Republic. ${ }^{2}$ Department of Immunology and Allergology, Faculty Hospital in Pilsen, Pilsen, Czech Republic. ${ }^{3}$ Department of Dermatovenerology, Faculty Hospital in Pilsen, Pilsen, Czech Republic.

${ }^{4}$ The National Institute of Public Health, Prague, Czech Republic

\section{Acknowledgements}

Support to this article has been provided by the EAACI National Society Committee Support Initiative. The authors wish to thank the EAACI NASC for that.

\section{Competing interests}

The authors declare that they have no competing interests.

\section{Availability of data and materials}

Anonymized source data used for the statistical analysis are available at the Department of Immunology and Allergology of the Faculty of Medicine in Pilsen. These data are available from the corresponding author on reasonable request.

\section{Consent for publication}

Not applicable.

\section{Ethics approval and consent to participate}

The study was approved by the Ethics Committee of the Faculty Hospital in Pilsen on 6th October 2016 with reference number 404/2016. Data have been anonymized before the analysis.

\section{Funding}

The study was funded from internal sources of Faculty of Medicine in Pilsen and Faculty Hospital in Pilsen. No funding was received from any external sources.

\section{Publisher's Note}

Springer Nature remains neutral with regard to jurisdictional claims in published maps and institutional affiliations.

Received: 6 December 2017 Accepted: 23 April 2018

Published online: 04 June 2018

\section{References}

1. Banerjee S, Resch Y, Chen KW, Swoboda I, Focke-Tejkl M, Blatt K, et al. Der p 11 is a major allergen for house dust mite-allergic patients suffering from atopic dermatitis. J Invest Dermatol. 2015;135(1):102-9.

2. Resch Y, Michel S, Kabesch M, Lupinek C, Valenta R, Vrtala S. Different IgE recognition of mite allergen components in asthmatic and nonasthmatic children. J Allergy Clin Immunol. 2015;136(4):1083-91.

3. Custovic A, Sonntag HJ, Buchan IE, Belgrave D, Simpson A, Prosperi MC Evolution pathways of IgE responses to grass and mite allergens throughout childhood. J Allergy Clin Immunol. 2015;136(6):1645-52.

4. Kidon MI, Chiang WC, Liew WK, Ong TC, Tiong YS, Wong KN, et al. Mite component-specific lgE repertoire and phenotypes of allergic disease in childhood: the tropical perspective. Pediatr Allergy Immunol. 2011;22(2):202-10.

5. Bousquet PJ, Chinn S, Janson C, Kogevinas M, Burney P, Jarvis D, et al. Geographical variation in the prevalence of positive skin tests to environmental aeroallergens in the European Community Respiratory Health Survey I. Allergy. 2007;62(3):301-9.

6. Panzner P, Vachova M, Vitovcova P, Brodska P, Vlas T. A comprehensive analysis of middle-European molecular sensitization profiles to pollen allergens. Int Arch Allergy Immunol. 2014;164(1):74-82.
7. Stemeseder T, Klinglmayr E, Moser S, Lueftenegger L, Lang R, Himly M et al. Cross-sectional study on allergic sensitization of Austrian adolescents using molecule-based lgE profiling. Allergy. 2017;72(5):754-63.

8. Pomes A, Arruda LK. Investigating cockroach allergens: aiming to improve diagnosis and treatment of cockroach allergic patients. Methods. 2014;66(1):75-85.

9. Casset A, Mari A, Purohit A, Resch Y, Weghofer M, Ferrara R, et al. Varying allergen composition and content affects the in vivo allergenic activity of commercial Dermatophagoides pteronyssinus extracts. Int Arch Allergy Immunol. 2012;159(3):253-62.

10. Takai T, Okamoto Y, Okubo K, Nagata M, Sakaguchi M, Fukutomi Y, et al. Japanese Society of Allergology task force report on standardization of house dust mite allergen vaccines - secondary publication. Allergol Int. 2015;64(2):181-6.

11. Brunetto $B$, Tinghino $R$, Braschi MC, Antonicelli L, Pini C, lacovacci $P$. Characterization and comparison of commercially available mite extracts for in vivo diagnosis. Allergy. 2010;65(2):184-90.

12. Melioli G, Bonifazi F, Bonini S, Maggi E, Mussap M, Passalacqua G, et al. The ImmunoCAP ISAC molecular allergology approach in adult multisensitized Italian patients with respiratory symptoms. Clin Biochem. 2011;44(12):1005-11.

13. Huss-Marp J, Gutermuth J, Schaffner I, Darsow U, Pfab F, Brockow K, et al. Comparison of molecular and extract-based allergy diagnostics with multiplex and singleplex analysis. Allergo J Int. 2015;24:46-53.

14. Vandenbroucke JP, von Elm E, Altman DG, Gotzsche PC, Mulrow CD, Pocock SJ, et al. Strengthening the Reporting of Observational Studies in Epidemiology (STROBE): explanation and elaboration. PLoS Med. 2007;4(10):e297.

15. Barbosa MC, Santos AB, Ferriani VP, Pomes A, Chapman MD, Arruda LK. Efficacy of recombinant allergens for diagnosis of cockroach allergy in patients with asthma and/or rhinitis. Int Arch Allergy Immunol. 2013;161(3):213-9.

16. Arruda LK, Barbosa MC, Santos AB, Moreno AS, Chapman MD, Pomes A. Recombinant allergens for diagnosis of cockroach allergy. Curr Allergy Asthma Rep. 2014;14(4):428.

17. Scala E, Alessandri C, Bernardi ML, Ferrara R, Palazzo P, Pomponi D, et al. Cross-sectional survey on immunoglobulin E reactivity in 23,077 subjects using an allergenic molecule-based microarray detection system. Clin Exp Allergy. 2010;40(6):911-21.

18. Barber D, Arias J, Boquete M, Cardona V, Carrillo T, Gala G, et al. Analysis of mite allergic patients in a diverse territory by improved diagnostic tools. Clin Exp Allergy. 2012;42(7):1129-38.

19. Melioli G, Marcomini L, Agazzi A, Bazurro G, Tosca M, Rossi GA, et al. The IgE repertoire in children and adolescents resolved at component level: a cross-sectional study. Pediatr Allergy Immunol. 2012;23(5):433-40.

20. Westritschnig K, Sibanda E, Thomas W, Auer H, Aspock H, Pittner G, et al. Analysis of the sensitization profile towards allergens in central Africa. Clin Exp Allergy. 2003;33(1):22-7.

21. Pittner G, Vrtala S, Thomas WR, Weghofer M, Kundi M, Horak F, et al. Component-resolved diagnosis of house-dust mite allergy with purified natural and recombinant mite allergens. Clin Exp Allergy. 2004;34(4):597-603.

22. Thomas WR. House dust allergy and immunotherapy. Hum Vaccin Immunother. 2012:8(10):1469-78.

23. Hales BJ, Elliot CE, Chai LY, Pearce LJ, Tipayanon T, Hazell L, et al. Quantitation of IgE binding to the chitinase and chitinase-like house dust mite allergens Der p 15 and Der p 18 compared to the major and mid-range allergens. Int Arch Allergy Immunol. 2013;160(3):233-40.

24. Weghofer M, Grote M, Resch Y, Casset A, Kneidinger M, Kopec J, et al. Identification of Der p 23, a peritrophin-like protein, as a new major Dermatophagoides pteronyssinus allergen associated with the peritrophic matrix of mite fecal pellets. J Immunol. 2013;190(7):3059-67.

25. Thomas WR. Hierarchy and molecular properties of house dust mite allergens. Allergol Int. 2015;64(4):304-11.

26. Posa D, Perna S, Resch Y, Lupinek C, Panetta V, Hofmaier $S$, et al. Evolution and predictive value of IgE responses toward a comprehensive panel of house dust mite allergens during the first 2 decades of life. J Allergy Clin Immunol. 2017;139(2):541-9.

27. Takai T, Ikeda S. Barrier dysfunction caused by environmental proteases in the pathogenesis of allergic diseases. Allergol Int. 2011;60(1):25-35.

28. Renand A, Archila LD, McGinty J, Wambre E, Robinson D, Hales BJ, et al. Chronic cat allergen exposure induces a TH2 cell-dependent 
IgG4 response related to low sensitization. J Allergy Clin Immunol. 2015;136(6):1627-35.

29. Thomas WR. Geography of house dust mite allergens. Asian Pac J Allergy Immunol. 2010;28(4):211-24.

30. Simpson A, Green R, Custovic A, Woodcock A, Arruda LK, Chapman MD. Skin test reactivity to natural and recombinant Blomia and Dermatophagoides spp. allergens among mite allergic patients in the UK. Allergy. 2003;58(1):53-6.

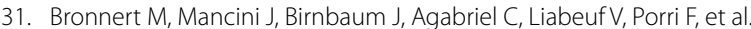
Component-resolved diagnosis with commercially available D. pteronyssinus Der p 1, Der $\mathrm{p} 2$ and Der p 10: relevant markers for house dust mite allergy. Clin Exp Allergy. 2012;42(9):1406-15.

32. Ayuso R, Reese G, Leong-Kee S, Plante M, Lehrer SB. Molecular basis of arthropod cross-reactivity: IgE-binding cross-reactive epitopes of shrimp, house dust mite and cockroach tropomyosins. Int Arch Allergy Immunol. 2002;129(1):38-48.

33. Hales BJ, Martin AC, Pearce LJ, Laing IA, Hayden CM, Goldblatt J, et al. IgE and IgG anti-house dust mite specificities in allergic disease. J Allergy Clin Immunol. 2006;118(2):361-7.
34. Satinover SM, Reefer AJ, Pomes A, Chapman MD, Platts-Mills TAE, Woodfolk JA. Specific IgE and lgG antibody-binding patterns to recombinant cockroach allergens. J Allergy Clin Immunol. 2005;115(4):803-9.

35. Resch Y, Weghofer M, Seiberler S, Horak F, Scheiblhofer S, Linhart B, et al. Molecular characterization of Der $\mathrm{p}$ 10: a diagnostic marker for broad sensitization in house dust mite allergy. Clin Exp Allergy. 2011:41(10):1468-77.

36. Mueller GA, Randall TA, Glesner J, Pedersen LC, Perera L, Edwards LL, et al. Serological, genomic and structural analyses of the major mite allergen Der p 23. Clin Exp Allergy. 2016;46(2):365-76.

37. Batard T, Baron-Bodo V, Martelet A, Le Mignon M, Lemoine $\mathrm{P}$, Jain $\mathrm{K}$, et al. Patterns of IgE sensitization in house dust mite-allergic patients: implications for allergen immunotherapy. Allergy. 2016;71(2):220-9.

38. Calderon M, Casale T, Nelson H, Demoly P. An evidence-based analysis of house dust mite allergen immunotherapy: a call for more rigorous clinical studies. J Allergy Clin Immunol. 2013;132(6):1322-36.
Ready to submit your research? Choose BMC and benefit from:

- fast, convenient online submission

- thorough peer review by experienced researchers in your field

- rapid publication on acceptance

- support for research data, including large and complex data types

- gold Open Access which fosters wider collaboration and increased citations

- maximum visibility for your research: over $100 \mathrm{M}$ website views per year

At $\mathrm{BMC}$, research is always in progress.

Learn more biomedcentral.com/submissions 